Meta

Journal des traducteurs

Translators' Journal

\title{
Arles, capitale française de la traduction littéraire
}

\section{Jean-Paul Partensky}

Volume 31, numéro 2, juin 1986

URI : https://id.erudit.org/iderudit/003792ar

DOI : https://doi.org/10.7202/003792ar

Aller au sommaire du numéro

Éditeur(s)

Les Presses de l'Université de Montréal

ISSN

0026-0452 (imprimé)

1492-1421 (numérique)

Découvrir la revue

Citer cette note

Partensky, J.-P. (1986). Arles, capitale française de la traduction littéraire. Meta, 31(2), 217-220. https://doi.org/10.7202/003792ar d'utilisation que vous pouvez consulter en ligne.

https://apropos.erudit.org/fr/usagers/politique-dutilisation/ 
BLOC-NOTES

\section{ARLES, CAPITALE FRANÇAISE DE LA TRADUCTION LITTÉRAIRE}

Les secondes Assises de la traduction littéraire (ATLAS) en Arles se sont tenues du 8 au 11 avril. M. Jean-Pierre Camoin, le maire d'Arles, a présidé la séance d'ouverture, au cours de laquelle M. Jean Gattegno, directeur du livre et de la lecture, a parlé du Grand prix national de traduction littéraire qui a été attribué cette année à Michel Leyris. Mine Laure Bataillon, présidente de l'ATLAS et de l'Asssociation des traducteurs littéraires de France, a fait part à son auditoire des réalisations du Collège international des traducteurs de Straelen en République fédérale d'Allemagne, objet d'une exposition dans le hall de l'hôtel de ville, ajoutant que celui d'Arles, qui ouvrira ses portes dès 1986, aura pour but :

1. d'assurer l'accueil et de permettre la rencontre de traducteurs littéraires de toutes nationalités, d'effectuer des travaux de recherche dont le résultat constituera un fonds de consultation pour le collège ; enfin, de promouvoir la traduction d'œuvres littéraires à l'étranger et celle d'cuvres étrangères en France :

2. d'assurer un travail de recherche bibliographique et lexicographique informatisé, enfin, de mettre en place une bibliothèque spécialisée (dictionnaires et encyclopédies, fiches de documentation) accessible à tous les organismes qui s'intéressent à la traduction.

Ce collège sera aménagé dans l'ancien hôpital où Van Gogh fut interné après s'être coupé l'oreille.

Près de 300 traducteurs, traductrices, linguis tes et professeurs venus pour la plupart d'Europe, mais aussi du Québec, s'étaient donné rendez-vous dans la ville d'Hubert Nyssen, à la fois auteur, traducteur et éditeur, qui a réservé une place très importante (dix-huit langues) aux traductions parmi les cuvres qui figurent dans son catalogue. Les auteurs anglophones y côtoient les romanciers d'Amérique latine, les écrivains allemands, nordiques mais aussi chinois et malais. Les Québécois et les romanciers contemporains du Canada anglais en sont absents, mais peut-être pas pour longtemps car Hubert Nyssen a les idées larges et se déclare prêt à faire une ex- périence : il nous incombe donc de le convaincre. Cet éditeur croit, en effet, que le traducteur doit être un découvreur avide de faire partager son enthousiasme pour un auteur étranger aux lecteurs de sa langue.

Les Assises proprement dites ont débuté par un débat entre linguistes et traducteurs sur " Les partís pris de traduction ", qui a été présidé par Hubert Nyssen.

Céline Zins l'a d'abord cerné en indiquant les trois grandes conceptions de la traduction littéraire actuellement envisagées :

1. La traduction ethnocentrique, très francisée, qu'elle a assimilée à une vision romantique des choses. Pour l'adepte de cette conception, il faut transposer les iées compte tenu de la culture des lecteurs de la langue dans laquelle il traduit. Sa traduction est très libre, c'est souvent une adaptation. Il laisse s'épancher sa propre subjectivité, si bien qu'il fait parfois ; une "belle infidèle" comme Baudelaire traduisant les Histoires extraordinaires d'Edgar Poe, et Vialatte traduisant Kafka.

2. La traduction littéraliste, "au plus près", pour reprendre un terme de marine, dont le défenseur le plus en vue est Antoine Berman. Selon les tenants de la littéralité, la fidélité est la qualité première du traducteur qui doit rechercher l'exactitude avant la beauté. Toutefois, comme l'a fait remarquer Inès Oseki-Dépré, il ne faut tout de même pas confondre ce type de traduction avec la traduction mot à mot qui est impossible car incompréhensible. Il s'agit toutefois d'une traduction qui n'hésite pas à bouleverser les structures et le vocabulaire de la langue d'arrivée.

3. Enfin, à mi-chemin entre ces deux pôles, la traduction préconisée par les tenants de la « littérarité ", subjective certes, présentant toutes les caractéristiques d'une oeuvre littéraire, mais qui est néanmoins fidèle à l'esprit sinon toujours à la lettre.

Les tenants de littérarité cherchent à rendre un texte en utilisant divers moyens pour transmettre aux lecteurs les particularités culturelles ou stylistiques du texte qu'ils traduisent. Il n'hésitent pas à bouleverser occasionnellement la syntaxe, à recourir à des emprunts ou à d'autres procédés afin de donner aux lecteurs les indices voulus pour situer le texte 
traduit par rapport à la langue de l'auteur et dans son contexte culturel.

C'est là une approche séduisante, adaptée à notre époque où la multiplication des moyens de communication met désormais à la portée du public maintes notions qui lui étaient auparavant étrangères.

Céline Zins se tournant vers les linguistes leur a demandé si les traductions étaient théorisables.

Ces derniers ont été unanimes à déclarer avec Jacqueline Guillermin que la traduction les intéressait non pas pour chercher des moyens destinés à aider les traducteurs à faire leur métier, mais plutôt parce que les traductions sont pour eux un matériau. L'analyse du fruit de nos travaux leur semble intéressante car elle alimente leurs théories.

Pour Marina Yaguello, s'il est vrai que tout ce que l'on a dit sur l'intraduisibilité a été battu en brèche, le traducteur a intérêt à se familiariser avec la linguistique pour comprendre ce qu'il fait. Il doit notamment savoir qu'il existe des dialectes, des sociolectes, etc.

Selon Henri Meschonnic, les traducteurs méprisent à tort la théorie car ils en font sans le savoir : une traduction révèle, en effet, toutes les idées sur la langue de son auteur. À son avis, en voulant être modeste, transparent, le traducteur se fourvoie : " l'obstacle à surmonter, c'est de concevoir la traduction comme une partie de la littérature".

Bernard Lortholary a déclaré que le parti pris "littéraliste", c'est-à-dire celui de l'auteur, " est un bateau fantôme qui n'aborde nulle part ". Et il a illustré son point de vue en citant le cas d'un traducteur qui a traduit un auteur grec en allemand en malmenant tant le vocabulaire et la syntaxe de cette langue par souci de fidélité qu'en définitive seuls ceux qui connaissent le grec peuvent comprendre sa traduction! Il ne faut pas non plus verser dans les excès francisants de Vialatte traduisant Kafka car finalement ses partis se sont retournés contre le lecteur, si bien que Bernard Lortholary a jugé bon de retraduire fidèlement Kafka, mais en prenant néanmoins le parti du lecteur.

Albert Bensoussan, partisan de la transparence, a souligné en tant que traducteur d'œuvres latino-américaines qu'il est nécessaire d'introduire en français des éléments un peu barbares pour marquer une différence entre la langue de Madrid et celle du Pérou, du Mexique, etc.

Georges Kasaï a signalé que les premières traductions de Freud étaient de "belles infidèles " car les traducteurs français ont voulu cartésianiser un auteur qui parle de l'inconscient, lequel n'est pas cartésien. Certains textes ont été traduits dix-sept fois. Et l'on a atteint des sommets dans l'exégèse puisque l'on a vu un texte de 10 pages accompagné de 50 pages de notes.

Pour terminer ce compte rendu sur la table ronde, il faut faire état d'une remarque très pertinente de Céline Zins qui a souligné qu'elle prenait toujours le parti non de l'auteur mais de sa langue en tant qu'écrivain par rapport à sa langue maternelle, idée qu'Inès Oseki-Depré a renforcée en disant : "Il faut se demander qu'est-ce que la lettre? La lettre, c'est le texte. Il vaut mieux parler de la lettre ou du texte que de l'auteur."

Enfin, Henri Meschonnic a fait remarquer que l'opposition entre parti pris pour l'auteur et parti pris pour le lecteur n'est pas valable car le lecteur est une multiplicité. Il a ajouté que la notion de littéralisme n'est pas efficace mais polémique et que celle de littérarité est meilleure.

À la suite de ce débat, je me suis élevé contre une adepte de la littéralité qui avait déclaré ne pas traduire pour ses lecteurs et ne pas hésiter à les faire souffrir lorsqu'ils devaient la suivre dans les méandres d'une pensée ardue.

À mon avis, le traducteur doit se préoccuper de son lecteur qui est le destinataire de son message. Il doit le faire, selon l'expression de Maurice-Edgar Coindreau, en singeant l'auteur, en trouvant les artifices voulus pour donner au lecteur une idée des sonorités ou des réalités culturelles sous-jacentes au texte de départ, notamment quand la langue d'arrivée n'offre pas les mêmes possibilités que la langue de départ. J'ai pris l'exemple des hispanismes que l'on pouvait utiliser pour rendre une certaine couleur dans Platero y yo. Et j'ai ajouté que si un auteur comme Thomas Raucat s'est permis d'utiliser toutes sortes de circonlocutions dans l'Honorable partie de campagne pour rendre la cocasserie des mours japonaises et les aspects laudatifs de la langue nippone, je ne voyais pas pourquoi je me priverais, moi traducteur, d'user de tels moyens. Pour ma part, je m'applique à mimer l'auteur, à me couler dans ses personnages et à multiplier mes registres.

Cette position m'a permis de faire la connaissance de Christian Guidicelli, qui a recueilli les propos de Coindreau publiés à la NRF sous le titre les Mémoires d'un traducteur et de Roger Vrigny, l'animateur des "Matinées litttéraires" sur FranceCulture. Ce dernier m'a invité à participer à une émission en compagnie d'Uffe Harder et de CarlGustav Bjurström, respectivement traducteurs en danois et en suédois de Claude Simon, lauréat du prix Nobel de littérature de Bernard Lortholary, linguiste et germaniste qui a traduit une quarantaine d'ouvrages, et de Jacques Lacarrière, écrivain qui s'est distingué par ses traductions du Grec Costas Taktsis. Nous nous sommes entretenus des rapports de la traduction et de la linguistique. La plupart estimant que la linguistique ne leur était d'aucun secours dans l'exercice de leur profession (les linguistes euxmêmes ayant reconnu la veille que la traduction était pour eux plus une matière à étudier qu'un champ d'application de leurs théories). J'ai alors indiqué que la Stylistique comparée de l'anglais et du français de Vinay et Darbelnet me paraît un ouvrage utile au traducteur qui permet de trouver des solutions à certains de ses problèmes, mais aussi fournit des éléments objectifs pour évaluer les traductions. Mais le plus intéressant, c'est que nous nous sommes attaqués au mythe selon lequel un grand auteur doit tou- 
jours être traduit par la même personne. Assimilant la traduction à l'intégration d'une ceuvre musicale, nous avons au contraire souligné que les lecteurs y gagneraient s'ils pouvaient choisir entre plusieurs versions. Par ailleurs, la collaboration pour la traduction d'une ceuvre peut être avantageuse car deux têtes valent mieux qu'une.

L'après-midi du 9 novembre a été consacré au thème "Claude Simon et ses traducteurs eurropéens".

Claude Simon, dont les cuvres ont été traduites en dix-huit langues, fut appelé à lire deux extraits particulièrement épineux, l'un de la Bataille de Pharsale et l'autre des Géorgiques repris successivement en suédois par Carl-Gustav Bjurström, en anglais par John Fletcher de Grande-Bretagne, en danois par Uffe Harder, en finnois par Jukka Mannerkorpi, en italien par Guido Neri, et en allemand par Helmut Scheffel. Ces traducteurs ont ensuite été invités à sigraaler à l'auditoire quels avaient été les principaux problèmes qu'ils avaient rencontrés dans ces extraits et à lui expliquer comment ils les avaient résolus.

Les difficultés sont nombreuses. Comme l'a fait remarquer Helmut Scheffel, rien que des "perceptions visuelles, mais à chaque instant, l'instant passé est souvenir, et l'instant futur est annoncé ". L'abondance des participes présents chez Simon a géné ses traducteurs qui ont dû soit en supprimer, scit les remplacer par des infinitifs, comme Neri.

L'écriture parfois phonétique de Simon a gêné Bjurström car le suédois s'écrit comme il se prononce. Il a dû ajouter des mots scatologiques pour rendre "sept heures et demie de caca de con science" et à des équivalences globales décalées. Le traducteur italien a surtout été frappé par le rythme à deux, puis trois temps du texte. Il a eu la sensation d'être transporté par une houle. John Fletcher a signalé que la langue anglaise se prête à la prose simonienne. Il a éprouvé le besoin d'étoffer, soi-disant, pour atténuer l'impact des mots vulgaires, c'est ainsi qu'il a traduit " la chair des femmes" par " the bare flesh of women bodies ", mais je me demande s'il n'a pas inconsciemment accru ce dernier.

Le traducteur finlandais Jukka Mannerkorpi a été obligé d'inverser des phrases et a mis, comme l'italien, l'accent sur la respiration, sur le rythme, de la phrase de Claude Simon " qu'il faut absolument rendre", ainsi que sur la nécessité de "faire vibrer chez le lecteur de la traduction les mêmes cordes que chez le lecteur du texte original ".

La journée du 10 novembre a débuté par des ateliers de traduction. J'ai assisté à l'atelier animé par Albert Bensoussan sur le dialogue dans la traduction de l'espagnol en français.

Les hispanisants sont arrivés aux conclusions suivantes :

- L'oralité est ce qui vieillit le plus vite.

- La traduction des dialogues suppose que le traducteur fasse corps avec ses personnages au point de devoir parfois les mimer devant la glace, comme une traductrice de Titiana Milliex. La traduction est d'ailleurs un exercice dangereux : quand les personnages sont dépressifs, les traducteurs le deviennent parfois.

- Les pronoms personnels que l'on emploie en français alourdissent souvent le texte dans la langue d'arrivée car l'espagnol s'en dispense : "dijo» se traduit par "dit-il ", " contesto", par " répondit-il", etc., d'où la nécessité, parfois, de supprimer ces verbes.

- Au nom de la transparence, le traducteur doit s'abstenir de faire des notes qui coupent inutilement la lecture. Il peut se permettre néanmoins d'ajouter en cas d'emprunt ou pour expliciter une réalité inconnue du lecteur, des incises, du genre : "le maguey, cet agave qui darde çà et là ses feuilles épineuses dans les prés mexicains, etc."

- Quand des auteurs latino-américains font des exercices comme écrire en espagnol en utilisant la syntaxe du guarani, le traducteur ne peut le suivre pas à pas sous peine de perdre son lecteur, mais il lui est loisible d'utiliser ponctuellement le même procédé en français en se servant de tournures compréhensibles pour le lecteur, ou en édifiant un langage codé qu'il peut éventuellement décoder dans une préface du traducteur. Cette idée de la préface, qui a d'abord suscité beaucoup de réticences a été adoptée pour les cas de difficultés vraiment spéciales.

- Pour ce qui est des jurons et des gros mots comme "carajo", qui abondent dans la littérature latinoaméricaine, il est possible de ne pas toujours les traduire de la même façon et de moduler pour ne pas alourdir la version française.

L'après-midi fut consacré au thème "Deux auteurs grecs et leurs traducteurs français ".

Philippe Dracodaïdis a expliqué l'impression pénible qu'il a éprouvée en lisant la traduction de son cuvre en français : il ne se reconnaissait pas et fut tout étonné quand on lui dit qu'il avait de la chance d'être tombé sur un aussi bon traducteur que le sien. Ce dernier, Michel Volkovitch, a donné à son auditoire une idée de la difficulté de la traduction du grec. Cette langue évoque pour lui le français de la Renaissance car il se caractérise par une surabondance de mots. Il explique qu'il n'existe pas de dictionnaire digne de ce nom. Les amis grecs interrogés ne donnaient presque jamais la même définition des mots qui lui posaient des problèmes; aussi le traducteur dut-il " casser les pieds" de son auteur pour avoir des éclaircissements que ce dernier n'était pas toujours en mesure de lui donner.

Ce fut ensuite le tour de Costas Taktsis d'exprimer ses yues. Il s'est élevé contre la distinction entre le grec démotique, les dialectes et la langue savante, distinction, à son avis artificielle : «Pour moi, il y a le grec moderne et le grec qui va de l'Antiquité à nos jours."

L'auteur a ensuite lu un passage d'un de ses textes, et son traducteur, Jacques Lacarrière, qui est aussi un homme de radio, a lu de façon très vivante sa traduction. Ce dernier a parlé aussi de l'échange de correspondance qu'il a eu avec Taktsis sur les dif- 
ficultés du texte et cité une phrase intéressante car elle illustre bien une particularité du français : "Quelle regrettable façon vous avez, vous autres Français, de parler d'une manière et d'écrire d'une autre.»

Le dernier jour a été consacré à un débat sur «Le traducteur dans la société » au cours duquel j’ai présenté une communication sur "L'expérience du Canada francophone "précédée d'observations faites par David Homel sur la condition des traducteurs littéraires canadiens. Françoise Cartano a parlé du traducteur aujourd'hui en France, déplorant que l'àvaloir qui lui est versé lors de la signature du contrat ne soit presque jamais amorti. En fait, il faut plus de 30000 exemplaires vendus pour que le traducteur touche quelque chose, si bien que l'on arrive à une situation paradoxale : plus les traducteurs traduisent, plus ils s'endettent. Les tantièmes versés sont dérisoires : de 0,5 à $3 \%$. Heureusement, un code des usages suivis dans l'édition des traductions vise à compenser les insuffisances de la loi française de 1957. Un traducteur qui fait 1000 pages par an, ce qui est rare, gagne 13000 dollars. Dans le cadre du point : "Pour un statut du traducteur littéraire européen ", il a été décidé de créer une structure européenne et d'instituer un Conseil permanent des traducteurs de la CEE qui s'attache à harmoniser les statuts des traducteurs littéraires européens, à élaborer un code de déontologie et à fixer un tarif minimum.

Comme l'an dernier, les Assises se sont terminées à l'hôtel de ville par la remise des prix ATLAS junior sous forme de livres donnés aux lauréats.

JEAN-PAUl PARTENSKY, TRADUCTEUR, MONTRÉAL 